\title{
Impairment of Memory for Spatial Context in Schizophrenia
}

\author{
Lydia Rizzo and Jean-Marie Danion \\ Insitut National de la Santé et de la Recherche Médicalc
}

\author{
Martial Van Der Linden \\ Service de Neuropsychologie \\ Jean-Georges Rohmer \\ Institut National de la Santé et de la Recherche Médicale
}

\begin{abstract}
A group of 33 patients with schizophrenia were compared with control participants using a spatial memory task in which words were presented on locations of a grid. In the first part of the experiment, recognition of target information (words) was tested. In the second, 2 tasks of spatial location (contextual information) were given involving different sets of words placed in different locations: A location memory task (determining which word was in a particular spatial location) explored an associative form of spatial memory, and a relocation task (determining where a particular word was located) explored an associative and a nonassociative form of spatial memory. Patients were more impaired with regard to the location memory task than to the target recognition and relocation tasks. The impairment was negatively correlated with Stroop task performance. The results suggest that schizophrenia is associated with a spatial context memory deficit, which could be due to defective associations between target and spatial information. This deficit seemed to be related to frontal dysfunction.
\end{abstract}

It has recently been suggested that the long-term memory impairment associated with schizophrenia might be related to a memory impairment for contextual information (GrasVincendon et al., 1994; Schwartz, Deutsch, Cohen, Warden, \& Deutsch, 1991). In the long-term memory realm, contextual information is traditionally distinguished from target information. Contextual information is information that typically falls on the periphery of attention and concerns where and when a specific event took place (independent context, Baddeley, 1982) and how it was affected by meaningful interpretations (interactive context). Target information is information that falls at the focus of attention and concerns what occurred during the specific event.

The context-memory deficit hypothesis postulates that patients with schizophrenia store target information normally but cannot recognize this information because they cannot associate it with contextual information. It is supported by studies that investigated schizophrenia patients' memory using the distinction between explicit and implicit memory tasks. Patients with schizophrenia are impaired in explicit memory tasks such as recall and recognition, in which the instructions make direct reference to the context of the learning episode. In contrast, they perform normally, or almost normally, in implicit memory tasks; that is, tasks such as word-stem completion

Lydia Rizzo, Jean-Marie Danion, and Jean-Georges Rohmer, Psychopathologie et Pharmacologie de la Cognition, Institut National de la Santé et de la Recherche Médicale (INSERM 405), Strasbourg, France; Martial Van Der Linden, Service de Neuropsychologie, Liège, Belgium; Danielle Grangé, CNUSC, Montpellier, France.

This research was supported by a grant from the Association of European Psychiatrists.

Correspondence concerning this article should be addressed to Jean-Marie Danion, INSERM Unité 405, Hôpitaux Universitaires de Strasbourg, Département de Psychiatrie, 1 Place de 1'Hôpital, BP 426, 67091 Strasbourg Cedex, France.
(Gras-Vincendon et al., 1994; Schwartz, Rosse, \& Deutsch, 1993), pursuit rotor tasks (Huston \& Shakow, 1949; Kornetsky, Pettit, \& Wynne, 1959), and repeated solving of the Tower of Hanoi or of its variants (Goldberg, Saint-Cyr, \& Weinberger, 1990; Gras-Vincendon et al., 1994; Schmand, Brand, \& Kuipers, 1992), in which the instructions do not make reference to the context of the learning episode. Insofar as the notion of contextual information is pivotal to the distinction between explicit and implicit memory tasks, it has been inferred from this pattern of memory dysfunction that memory is impaired in patients with schizophrenia when contextual information is required and spared when contextual memory is not required.

According to this interpretation, contextual information could be the key factor that determines the impaired cognitive profile in schizophrenia. This interpretation is further supported by the demonstration that patients with schizophrenia are usually more impaired in recall than in recognition tasks (Bauman \& Murray, 1968; Calev, 1984; Goldberg, Weinberger, Pliskin, Berman, \& Podd, 1989; Koh, Kayton, \& Peterson, 1976; Nachmani \& Cohen, 1969). Indeed, recall performance depends to a greater extent than recognition performance on associating target and contextual information. A failure to associate target and contextual information is therefore likely to have a greater deleterious effect on recall than on recognition performance. In free recall, in which the participant is asked to recollect the circumstances specified by the task, the role of contextual information is crucial. In contrast, recognition has been postulated to involve two components: conscious recollection, an elaborative component that relies on specific contextual information, and familiarity, a component independent of contextual information (Mandler, 1980; Jacoby \& Dallas, 1981). Recognition performance will therefore be affected differently by a failure to associate target and contextual information, depending on the respective roles played by recollection and familiarity in each particular task. If recogni- 
tion relies mainly on familiarity, and if familiarity is intact as seems to be the case in patients with schizophrenia (Huron et al., 1995), there will be little or no effect on performance.

However, these arguments in favor of a context-memory deficit hypothesis are only indirect, and few studies have specifically investigated this hypothesis with appropriate concepts and methods. Most of them have reported an impairment of two different forms of contextual information: frequency monitoring, as assessed by a frequency monitoring task (Gold, Randolf, Carpenter, Goldberg, \& Weinberger, 1992; Gras-Vincendon et al., 1994), and temporal order information, as assessed by a recency discrimination task (Schwartz et al., 1991). Moreover, schizophrenia is associated with a reality monitoring impairment; that is, an inability to discriminate previously read from previously self-generated items (Bentall, Baker, \& Havers, 1991). However, one study (Shoqeirat \& Mayes, 1988) concluded that memory for spatial context and memory for temporal context, as assessed by a recency discrimination task, were not disproportionately poorer than recall and recognition.

These discrepancies may simply reflect the fact that because the different forms of context memory are likely to depend on somewhat different cognitive processes, they may be differentially affected by schizophrenia. But some discrepancies could also be explained by the methodological inadequacies of certain studies. Cognitive neuropsychological studies of patients with organic amnesia (Mayes, Meudell, \& McDonald, 1991; Shoqeirat \& Mayes, 1991) or frontal lobe lesions (Schacter, 1987) have shown that two main methodological constraints must be respected. First, memory for target information must be matched in the normal and patient groups, so the experiment can demonstrate that patients are morc impaired in tasks assessing contextual information memory than in ones assessing target information memory. In other words, the experiment must demonstrate a disproportionately severe memory deficit for contextual information. This is the only way to establish that a contextual memory impairment represents the fundamental deficit in patients and is the cause, rather than the consequence, of generally poor memory. Matching has usually been obtained by testing patients in less demanding conditions than controls, for instance by giving patients more opportunity to learn the material or by testing them after shorter delays.

An alternative procedure is to exclude from the analyses patients who perform too poorly in the target memory task. The logic of this matching procedure is provided by the two-component theories of recognition memory (Mandler, 1980; Jacoby \& Dallas, 1981), which postulate that recognition involves conscious recollection or familiarity or both, with the former, but not the latter, requiring the availability of contextual information. As previously discussed, some patients with schizophrenia are likely to perform normally, or almost normally, in a target recognition task inasmuch as the task does not rely heavily on contextual information. The second methodological constraint is related to the sensitivity of the tasks used for comparing target and contextual information memory. These two forms of memory must not be confounded with recall and recognition memory, inasmuch as these tasks are differentially sensitive to experimental and pathological situa- tions. In other words, it is essential that memory for target information and memory for contextual information are assessed by the same type of tasks, for instance, recognition tasks.

The present study, which investigated context memory for spatial information in schizophrenic patients, had two main goals. The first was to assess memory for spatial information relative to memory for target information. A modified version of the spatial memory task developed by Shoqcirat and Mayes (1991) and Smith and Milner (1981) was used. Participants were presented with a list of words, each word being placed on a particular location of a grid. Encoding of spatial locations (contextual information) was incidental, because participants were told to remember the words (target information). Testing was divided into two parts. In the first, recognition of the target information was tested. In the second, two different spatial location tasks were given, involving different sets of words placed in different locations. They were intended to contrast a nonassociative form of spatial memory, that is, spatial information memory per se, and an associative form of spatial memory, that is, memory for the association between target and contextual information. Target memory and context memory were assessed by three-alternative forced-choicc recognition tasks. Recognition matching across groups was obtained by cxcluding the patients who scored too poorly in the recognition task for target information. The context-memory deficit hypothesis predicts that, compared with control group participants, individuals with schizophrenia will show a disproportionate memory deficit for spatial location (context) relative to word (target) recognition. The sccond aim of the experiment was to examine whether spatial memory task performance was correlated with performance in tasks measuring the severity of the memory deficit and with performance in tasks sensitive to frontal lobe dysfunction. The context-memory deficit hypothesis predicts that the degree to which patients with schizophrenia show disproportionately severe deficit for context memory will correlate significantly with the severity of the memory deficit (Shoqeirat \& Mayes, 1991). Tests scnsitive to frontal lobe dysfunction were used to assess the part played by the frontal lobe dysfunction associated with schizophrenia (Weinberger, 1988; Winn, 1994) in spatial memory task performance.

\section{Method}

\section{Participants}

Thirty-three French-speaking patients ( 21 men, 12 women; mean age $=32.8$ years, $S D=8.2$; mean educational level $=10.2$ years, $S D=3.3$ ) participated in the study, which was part of a broader investigation of memory for different forms of contextual information. An investigation of temporal context memory in which these patients participated is reported elsewhere (Rizzo, Danion, Van Der Linden, \& Grangé, 1996). There were 5 inpatients and 28 outpatients. All fulfilled Diagnostic and Statistical Manual of Mental Disorders, third edition, revised (DSM-III-R; American Psychiatric Association, 1987) criteria for schizophrenia (mean duration of illness $=8.5$ years, $S D=5.3$ ). Patients with histories of traumatic brain injury, epilepsy, alcohol or substance abuse, other diagnosable neurological conditions or organic mental disorder, or who had been treated with antidepressants, benzodiazepines, or lithium were excluded. All patients were clinically stabilized. Thirty-one patients were receiving a long-term 
neuroleptic treatment, administered in a standard dose (mean dose $=296 \mathrm{mg}, S D=246$, of chlorpromazine or equivalent; Chouinard \& Denis, 1980). Sixteen were also receiving anti-Parkinsonian treatment (trihexyphenidyl, mean dose $=5.9 \mathrm{mg}, S D=3.8$; or tropatepine, mean dose $=11.4 \mathrm{mg}, S D=6.3$ ). Two patients were receiving no treatment. The normal group comprised 33 individuals ( 21 men, 12 women) previously matched with the 33 patients for sex, age ( $M=31.3$ years, $S D=8.7)$, and educational level $(M=11.2$ years, $S D=3.5)$. The two groups did not significantly differ in age $(t=0.75, n s)$ or education $(t=-1.12, n s)$. The control group had no history of alcoholism, drug abuse, or neurological or psychiatric illness and were not taking any drugs. All participants provided informed consent. Global psychiatric symptomatology was assessed by the Brief Psychiatric Rating Scale (Overall \& Gorham, 1962; $M=48.5, S D=14.7$ ). Positive and negative symptoms were assessed by the Scale for the Assessment of Positive Symptoms (Andreasen, 1983b; $M=34.7$, $S D=28.1$ ) and the Scale for the Assessment of Negative Symptoms (Andreasen, 1983a; $M=54.3, S D=25.7$ ). Extrapyramidal symptoms were assessed by the Simpson-Angus Scale (Simpson \& Angus, 1970); they were observed in 27 patients in very mild intensity $(M=5.3$, $S D=5.6$ ). Tardive dyskinesias were assessed by the Abnormal Involuntary Movement Scale (Guy, 1976); they were present in 18 patients, and their intensity was very mild $(M=3.1, S D=5.5)$.

Table 1 shows subjects' performance in tasks assessing 1Q, global memory competence, and memory spans and in tasks sensitive to frontal lobe dysfunction. IQ was assessed using a short version (Britton \& Savage, 1966; Crawford, Allan, \& Jack, 1992) of the Wechsler Adult Intelligence Scale-Revised (WAIS-R), verbal IQ was measured using vocabulary and comprehension subtests, and performance IQ using block design and picture arrangement subtests. Global memory competence was assessed using the Wechsler Memory Scale-Revised
(WMS-R 1980, 1989; Wechsler, 1981) and the Rivermead Behavioural Memory Test (RBMT; Baddeley, 1987; Wilson, Cockburn, Baddeley, \& Hiorns, 1989), selected because it provides a more ecological assessment of memory competence. In all these tasks, performance was significantly lower in the schizophrenic than in the control group $(t \mathrm{~s} \geq 3.81, p<.0001)$.

Memory spans included a spatial span, as assessed by the Block Tapping Test (Milner, 1971), a letter span, and a visuopattern span (Philips, 1983; Wilson, Scott, \& Power, 1987). In order to measure the visuopattern span, subjects were presented with a visual pattern made up of little boxes, some of which were black. The pattern was displayed for two seconds. After a blank interval, subjects drew a cross on a grid to indicate the places where the black boxes had been. The patterns were simple at first but subsequently became more complicated. Memory spans were significantly lower in schizophrenic than in control subjects $(t \mathrm{~s} \geq 2.91, p \mathrm{~s}<.005)$.

Tasks sensitive to frontal lobe dysfunction included the Wisconsin Card Sorting Test (WCST modified; Nelson, 1976), the word fluency task (Bruyer \& Tuyumbu, 1981) and the Stroop Color and Word Test (Stroop, 1935). In this version, three words were used with their matching ink colors: red, green, and blue. Three conditions were compared: naming ink colors of solid color squares, reading color words in black ink, and naming ink colors of incompatible color words. Performance in the WCST and on the fluency task was significantly lower in the schizophrenic group ( $t \mathrm{~s} \geq 2.66, p \mathrm{~s}<.005$ ). This was also the case with Stroop task performance, which was analyzed using multivariate analyses of variance (MANOVAs) with condition as a repeated measure and group as a between-subjects factor. A MANOVA carried out on the time required to complete each condition revealed a significant condition, $F(2,63)=285, p<.0001$, and group effect, $F(1$, $64)=27.6, p<.001$, and a significant interaction between conditions

Table 1

Neuropsychological Assessment

\begin{tabular}{|c|c|c|c|c|c|}
\hline \multirow[b]{2}{*}{ Measure } & \multicolumn{2}{|c|}{$\begin{array}{l}\text { Schizophrenia } \\
\text { patients } \\
(n=33)\end{array}$} & \multicolumn{2}{|c|}{$\begin{array}{c}\text { Control } \\
\text { group } \\
(n=33)\end{array}$} & \multirow[b]{2}{*}{$t$} \\
\hline & $M$ & $S D$ & $M$ & $S D$ & \\
\hline \multicolumn{6}{|l|}{ WAIS-R } \\
\hline Verbal IQ & 91 & 22 & 110 & 19 & $-3.81^{* * *}$ \\
\hline Performance IQ & 78 & 16 & 106 & 14 & $-7.41^{* * *}$ \\
\hline Total IQ & 85 & 18 & 110 & 18 & $-5.59 * * *$ \\
\hline Wechsler Memory Scale Revised, global memory index & 78 & 22 & 109 & 15 & $-6.39^{* * *}$ \\
\hline Rivermead Behavioral Memory Test & 16 & 5 & 24 & 0.4 & $-7.45^{* * *}$ \\
\hline Letter span & 4.7 & 0.9 & 5.4 & 0.9 & $-2.91^{* *}$ \\
\hline Spatial span & 4.1 & 0.9 & 5.8 & 1.1 & $-6.61 * * *$ \\
\hline Visuopattern span & 6.2 & 1.9 & 8.8 & 1.3 & $-6.33^{* * *}$ \\
\hline Word fluency: total number of correct responses & 26 & 11 & 41 & 10 & $-5.47^{m * *}$ \\
\hline \multicolumn{6}{|l|}{ WCST } \\
\hline Number of categories & 5.1 & 1.8 & 5.9 & 0.4 & $-2.66^{* *}$ \\
\hline Number of errors & 4.8 & 6.4 & 0.2 & 0.8 & $3.97^{* * *}$ \\
\hline Number of perseverative errors & 2.8 & 3.4 & 0.03 & 0.2 & $4.53^{* * *}$ \\
\hline \multicolumn{6}{|l|}{ Stroop Color and Word Test: } \\
\hline \multicolumn{6}{|l|}{ Ink colors naming } \\
\hline Total time $(\mathrm{s})$ & 78 & 22.5 & 57 & 9.5 & $5.01^{* * *}$ \\
\hline Number of total errors & 2 & 1.5 & 0.1 & 0.5 & $6.19^{* * *}$ \\
\hline \multicolumn{6}{|l|}{ Words reading } \\
\hline Total time (s) & 53 & 14.5 & 40 & 5.7 & $4.62 * * *$ \\
\hline Number of total errors & 0.3 & 0.6 & 0.03 & 0.2 & $2.32^{*}$ \\
\hline \multicolumn{6}{|l|}{ Color naming } \\
\hline Total time (s) & 144 & 39 & 109.6 & 22 & $4.35^{* * * *}$ \\
\hline Number of total errors & 5.6 & 5 & 2 & 2.7 & $3.42^{* * *}$ \\
\hline
\end{tabular}

Note. WAIS-R $=$ Wechsler Adult Intelligence Scale-Revised; WCST $=$ Wisconsin Card Sorting Test. Statistical comparisons are by Student's $t$ tests.

${ }^{*} p<.05 .{ }^{* *} p<.005 .{ }^{* * *} p<.0001$. 
and groups, $F(2,63)=6.95, p<.0001$. A MANOVA carried out on the number of noncorrected errors in each condition also revealed a significant condition effect, $F(2,63)=30.5, p<.0001$, and group effect, $F(1,64)=11.8, p<.001$, and a significant interaction between condition and group, $F(2,64)=4.68, p<.02$. A MANOVA carried out on the total number of errors yielded similar results. Additional analyses showed that interactions between conditions and groups were due to a significantly more severe impairment of schizophrenic patients in the interference condition, that is, naming ink color of incompatible color words, than in the other two conditions, compared with controls (all $p \mathrm{~s}<.05$ ), with the exception of the total number of errors in the naming ink color of squares, $F(1,64)=3.64, p=.06$.

\section{Memory for Target and Spatial Information}

Materials and experimental procedure were elaborated on the basis of pilot studies. They were intended to elicit in nonschizophrenic participants high, subceiling, target information recognition levels of around $80-90 \%$, and intermediate contextual information recognition levels of around $60-70 \%$. Each participant was tested individually in two sessions at least 1 day apart.

\section{Materials}

Three lists of 36 French words were constructed, each list being further divided into three sublists of 12 words. Words were nouns of 5 letters and of low frequency occurrence (mean frequency of occurrence: list $1=6.09$, list $2=6.76$, list $3=6.43 ; F=0.08, n s$ ). Two lists (a target and a distractor list) were used during the first session, the remaining list being used during the second session. Words were presented on a grid divided into $64(8 \times 8)$ rectangles. The presentation and testing of the words and locations were controlled by a microcomputer.

\section{Procedure}

Target and spatial context information memory were assessed during the first and second sessions, respectively. Contextual information memory was always tested during the second session to ensure that spatial information was encoded incidentally. During the first session, the 36 words belonging to one of the three lists were presented on the grid. Each word was presented for $2 \mathrm{~s}$, then disappeared and was followed $3 \mathrm{~s}$ later by the next word. Subjects were required to remember the words. No mention was made of the spatial location of the words.

The test phase began 5 min later. A three-alternative forced-choice recognition task was used to assess memory for target information. In this task, referred to as a target recognition task, subjects were presented with 12 series of 3 words, one of which, the target word, had been previously presented, the other two being distractors. The 12 target words belonged to one sublist from the previously presented list. The 24 distractor words belonged to two sublists from one of the non-previously presented lists. This was intended to ensure that the number of items presented during the study and test phases of the target information recognition task was identical to that in the contextual information recognition tasks carried out during the second session. Subjects were asked to recognize the words that had been previously presented.

During the study phase of the second session, the 36 words belonging to the not-yet-presented list were presented to the participants in exactly the same way as in the first session. The test phase comprised two tasks, the order of which was counterbalanced. They were three-alternative forced-choice recognition tasks:

1. One was a location memory task, which consisted of determining which word was in a particular spatial location. Subjects were presented with 12 series of three words, each of which belonged to a different sublist of the previously presented list. Each series of three words was presented successively below the grid, in which one location was colored black. Participants were asked to identify which of the three words had been located in the rectangle during the study phase. They could only answer on the basis of their ability to associate spatial contextual and target information, that is, on the basis of an associative spatial memory.

2. The other was a relocation task, which consisted in determining where a particular word was located. Participants were presented with 12 series of three black rectangles on the grid and one word below the grid. Words belonged to one of the three sublists of the previously presented list, but this sublist was different from the target sublist used in the spatial recognition task. Of the three rectangles presented in each series, one had been occupied by the word presented under the grid, and the others had not been occupied by any word during the learning phase. Participants were asked to identify which of the rectangles was occupied by the word during the study phase. They could answer on the basis of associations between target and spatial information. If these associations were defective, however, they could also answer on the basis of recognition memory whether or not locations were occupied by words during the study phase, irrespective of which particular words occupied these locations. In other words, when they had a defective associative spatial memory, participants could use nonassociative spatial memory to complete the relocation task. Such compensatory strategies would be expressed by a memory performance profile characterized by a disproportionate performance deficit in location memory, but not relocation, tasks. In both location memory and relocation tasks, the locations selected within each series were separated by at least four rectangles $(M \mathrm{~s}=5.9$ versus 6.1 , respectively, $n s$ ). Lists were rotated across sessions to ensure that, as far as possible, each list was presented the same number of times in each task. Sublists were rotated across tasks to ensure that, as far as possible, each sublist was presented the same number of times in each study and test phase. Lists and sublists were presented to a given patient and his or her matched control in an identical combination.

\section{Results}

The performance of all the participants in the schizophrenic and control groups in the target recognition, location memory, and relocation tasks is shown in Figure 1. The mean number of correct responses in the target recognition task was $10.8(90 \%)$ in the control group and $9.5(79 \%)$ in the schizophrenic group. These figures indicate that subceiling target information recognition levels were obtained. Performance of schizophrenic patients was lower than that of control subjects in the three tasks. The deficit was more marked in the location memory task than in the other two tasks: Location memory performance ( $M=4.4, S D=1.8$ correct responses) was close to chance performance (4.0). This was confirmed by a MANOVA with the number of correct responses in the three tasks as a repeated measurc and group as a between subject factor. There was a significant task effect, $F(2,63)=213, p<.0001$, and group effect, $F(1,64)=52.4, p<.0001$, and a significant interaction between task and group, $F(2,63)=4.24, p<.02$. Further analyses revealed that performance in the target recognition task was significantly higher than that in the location memory, $F(1,64)=252.4, p<.0001$, and relocation, $F(1,64)=303.2, p<.0001$, tasks, whereas performance in the two latter tasks was not significantly different, $F(1,64)=0.04$. Schizophrenia patients' performance was significantly im- 
paired in the three tasks, $t \mathrm{~s}(64) \geq 3.69, \mathrm{ps}<.001$. The interaction between task and group was duc to a significantly greater impairment of performance in the location memory than in the target recognition task in schizophrenic patients, $F(1,64)=8.61, p<.005$; schizophrenia patients' performance in the location memory task was also more impaired than in the relocation task, whereas the opposite pattern of performance was observed in control participants, but the interaction failed to reach significance, $F(1,64)=3.40, p=.07$. The interaction between relocation and target recognition tasks and groups was not significant, $F(1,64)=0.85$. Relocation performance of patients was significantly different from chance, $t(32)=3.26, p<.005$, but location memory performance was not, $t(32)=0.39, n s$. These analyses were also carried out after the exclusion of those who obtained a perfect score in the target recognition task ( 9 nonschizophrenic versus 4 schizophrenic participants, $\left.\chi^{2}=1.79, n s\right)$. They yielded similar conclusions (data not shown).

These results indicate that patients' location memory was disproportionately impaired relative to target recognition and, to a lesser extent, relocation and that this disproportionate impairment was not due to a ceiling effect in target recognition performance. However, they were obtained in conditions where memory for target information was not equalized in the patient and control groups and scalings effects cannot be excluded. A secondary analysis was therefore carried out on a subgroup of 24 schizophrenic patients and their 24 matched controls, that is, after the exclusion from the analysis of the 9 patients whose performance in the target recognition task was two standard deviations below that of control subjects. The mean target recognition, location memory, and relocation performance of these two subgroups are shown in Figure 2. This time, the target recognition performance of schizophrenic patients $(M=10.5, S D=1.0$, correct responses; $87.5 \%)$ was very similar to that of control participants $(M=10.7, S D=1.0$; $89 \%$ ), but patients still exhibited a memory defect as indicated by a significantly lower WMS-R global memory index $(M=82.7$, $S D=21.1$, versus $M=107.5, S D=16.2$, in the control group), $t(46)=-4.35, p<.0001$, and delayed recall score $(M=86.4$,

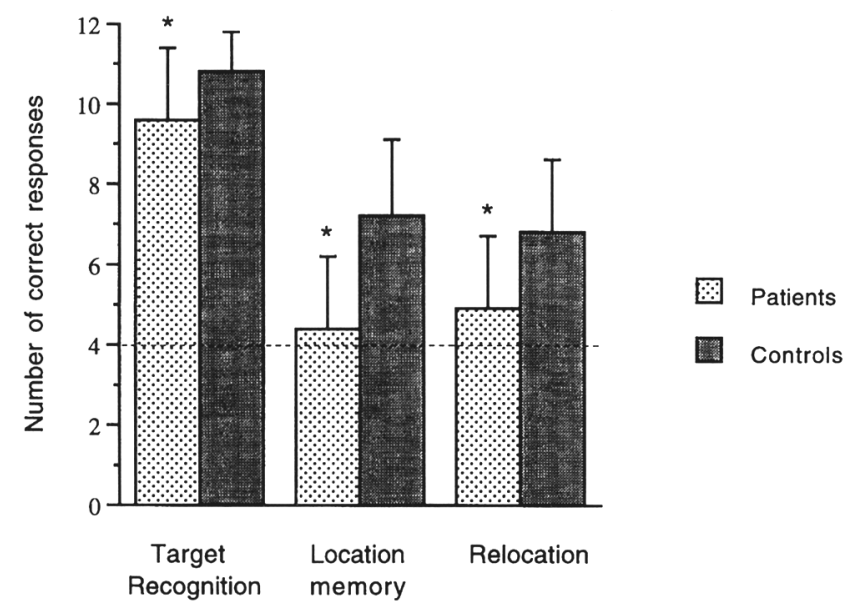

Figure 1. Spatial memory task performance $(M \pm S D)$ in the schizophrenia patient $(n=33)$ and control $(n=33)$ groups. Dotted line indicates chance performance. ${ }^{*} p<.001$ compared with controls.

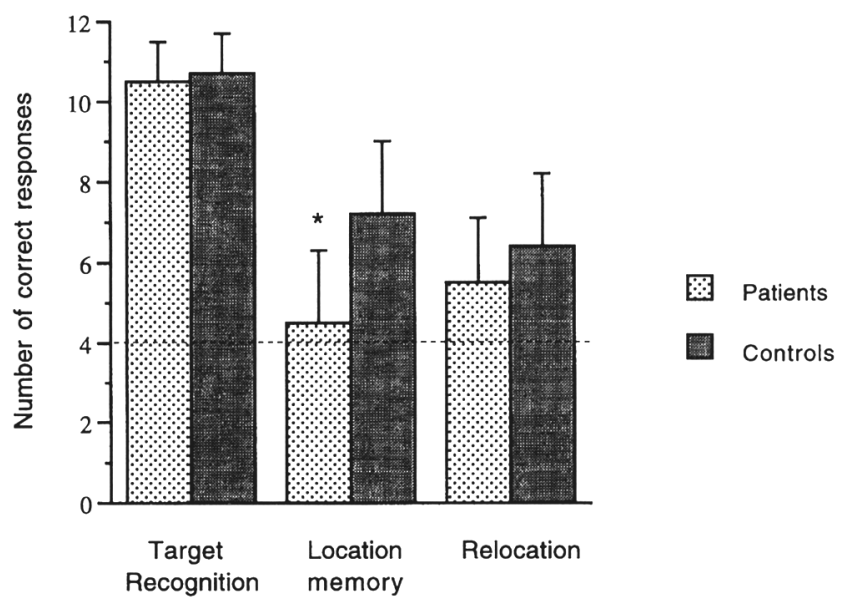

Figure 2. Spatial memory task performance $(M \pm S D)$ in the schizophrenia patient $(n=24)$ and control $(n=24)$ subgroups. Dotted line indicates chance performance. ${ }^{*} p<.001$ compared with controls.

$S D=18.1$, versus $M=108.4, S D=12.6$, in the control group), $t(46)=-4.64, p<.0001$. In contrast to target recognition performance, patients' location memory performance still remained close to chance $(M=4.5, S D=1.8$, versus $M=7.2$, $S D=1.8$, in controls). Patients' performance in the relocation task $(M=5.5, S D=1.6)$ was slightly lower than that of control participants $(M=6.4, S D=1.8)$. A MANOVA carried out on performance in the three tasks revealed a significant task, $F(2$, $45)=214, p<.0001$, and group effect, $F(1,46)=21.4, p<$ .0001 , and a significant interaction between task and group, $F(2,45)=8.01, p<.005$. Further analyses indicated that performance in the target recognition task was significantly higher than that in the location memory, $F(1,46)=271.2, p<$ .0001 , and relocation, $F(1,46)=273.4, p<.0001$, tasks, with performance in the last two tasks not being significantly different $(F=0.09)$. Schizophrenic patients' performance was only significantly lower than that of controls in the location memory task $t(46)=-5.01, p<.0001$. The interaction between tasks and groups was due to the fact that schizophrenic patients were disproportionately impaired in the location memory task, relative to the target recognition, $F(1$, $46)=16.4, p<.0005$, and the relocation, $F(1,46)=5.98, p<$ .02 , tasks. The interaction between relocation and target recognition tasks and groups was not significant $(F=1.23)$. Relocation performance was significantly different from chance, $t(23)=4.47, p<.002$, but location memory performance was not, $t(23)=1.46, n s$.

The 9 patients who were excluded from these secondary analyses were characterized by a severe memory deficit, as indicated by their significantly lower WMS-R global memory index $(M=64, S D=18$, versus $M=82, S D=21), t(31)=$ $2.25, p<.05$. Interestingly, they performed at chance in both location memory ( $M=4.0, S D=1.9$, correct responses) and relocation $(M=3.8, S D=1.7)$ tasks, but not in the target recognition task $(M=7.0, S D=0.9)$.

In order to evaluate the contribution of anticholinergic medication to memory deficit, patients were subdivided into those who were receiving anticholinergic medication $(n=16)$ and those who were not $(n=17)$. The performance of patients 
who were or were not receiving anticholinergic medication was not significantly different: target recognition performance ( $M=9.5, S D=1.9$, versus $M=9.5, S D=1.6$, respectively), $t(31)=0.5, n s ;$ location memory performance $(M=4.4$, $S D=1.8$, versus $M=4.3, S D=1.7$, respectively), $t(31)=$ $0.13, n s ;$ and relocation performance $(M=5.0, S D=1.6$, versus $M=5.0, S D=1.9), t \mathrm{~s}(31)=0.09, n s$.

\section{Correlation Analyses}

Pearson product-moment correlation coefficients between performance in the spatial memory tasks and age; measures of psychiatric and neurological symptomatology; memory spans; and performance in the WAIS-R, WMS-R, RBMT, and tasks sensitive to frontal lobe dysfunction were calculated. Pearson product-moment correlation coefficients between performance in the target recognition task and performance in the location memory and relocation tasks were also calculated. In vicw of the number of correlations calculated, only those that were significant in both the whole groups and the subgroups that excluded 9 subjects are reported.

Patients with schizophrenia. Performance in the spatial memory tasks was significantly correlated with the severity of the memory deficit; there were significant correlations between target recognition memory and the WMS-R global memory index (whole group, $r=.57$; subgroup, $r=.48$; $p \mathrm{~s}<.05)$, location memory performance and delayed recall performance of the WMS-R (whole group, $r=.47$; subgroup, $r=.52 ; p s<.05)$ and relocation performance and RBMT performance (whole group, $r=.59$; subgroup, $r=.63$; ps $<.02$ ). Turning to tasks sensitive to frontal lobe dysfunction, location memory performance was significantly and negatively correlated with performance in the interference condition of the Stroop task, as assessed by the number of corrected errors (whole group, $r=-.39$; subgroup, $r=-.55$; $p s<.05$ ) and noncorrected errors (whole group, $r=-.45$; subgroup, $r=-.54 ; p s<.05$ ). Finally, relocation performance was significantly correlated with the visuopattern span (whole group, $r=.57$; subgroup, $r=.57 ; p \mathrm{~s}<.005$ ). There were no correlations between performance in the target recognition, location memory, and relocation tasks, nor between any of these and IQ, age, age of onset and duration of schizophrenia, intensity of positive and negative symptoms, or neurological symptomatology.

Control participants. In the control group, the only statistically significant correlations of interest concerned performance in the relocation task and the visual memory subtest of the WMS-R $(r=.37, p<.05)$, and performance in the location memory and fluency tasks $(r=.50, p<.005)$.

Taken together, these correlation patterns suggest that performance in the location memory task was related to performance in some tasks sensitive to frontal lobe dysfunction (Stroop task in the schizophrenia group and fluency tasks in the control group) and that performance in the relocation task was related to performance in visuospatial tasks.

\section{Discussion}

The main finding of this study was that patients with schizophrenia were more impaired with regard to a location memory task than to target recognition and, to a lesser extent, relocation tasks. This result cannot be explained as an artifact arising from testing recognition memory for target and context information at different delays: Target and context information recognition were tested after the same delay, and the order of the two spatial tasks was counterbalanced. Nor can the result be explained in terms of differences in task sensitivity, because only variations in three-alternative forced-choice recognition tasks were used, with an identical number of items displayed during the study and test phases. The result cannot be explained in terms of ceiling effect, because the disproportionate performance deficit in the spatial memory task was still observed after the exclusion of the participants who obtained a perfect score in the target recognition task. It cannot be explained in terms of scaling effects, because the disproportionate performance deficit in the spatial memory task was observed not only in the whole group, but also in the subgroup of patients matched to controls on target recognition performance. Finally, because performance in the spatial memory tasks was significantly lower than in the target recognition task, it could be argued that the disproportionate impairment of location memory performance reflects differences in task difficulty. Howcver, differences in task difficulty cannot explain why the subgroup of matched patients was more impaired with regard to the location memory than to the relocation tasks; the overall location and relocation memory performance of subgroups of patients and of control participants was not significantly different, suggesting that the two tasks were of similar difficulty.

The finding of this study is at variance with that of a previous study (Shoqeirat \& Mayes, 1988), which did not observe any disproportionate spatial memory deficit using tasks of different scnsitivity (free and cued recall). But that study is hardly comparable to the present one, becausc it explored acute, rather than chronic, schizophrenia. The present results are likely to be relevant to the global and, possibly, everyday long-term memory deficit of schizophrenic patients. Indeed, as predicted by the context-memory deficit hypothesis, the disproportionate performance deficit obscrved in the location memory task was significantly correlated with the severity of the long-term memory deficit, as measured by the delayed recall score on the WMS-R, therefore validating a major prediction of the context-memory deficit hypothesis. Moreover, there was a significant correlation between relocation performance and memory competence as assessed by the RBMT, a memory task with ecological validity (Baddeley, 1987; Wilson, Cockburn, Baddeley, \& Hiorns, 1989).

It should be noted that the context-memory deficit hypothesis also predicts a significant correlation between contextual and target recognition memory. This study failed to find such a correlation. However, this failure can hardly call into question the role played by the contextual memory deficit in the global memory deficit of schizophrenic patients. As stated by Shoqeirat and Mayes (1991), the prediction is that there will be an overall association between the extent to which context memory is disrupted and the severity of the amnesia, whereas the relationship between any one specific kind of context memory and target recognition may be weak. This would probably be 
the case when performance in the target recognition task relied mainly on familiarity.

Before the pattern of memory dysfunction can be attributed to the illness, it is necessary to consider the potentially confounding role of treatments, because many of the patients were receiving a chronic neuroleptic treatment, and 16 of them, anticholinergic agents. Patients treated with benzodiazepines and antidepressants wcre excluded. It is unlikely that the memory impairment was due to anticholinergic agents, because memory performance was not significantly different in patients who were and those who were not receiving these drugs. Because all but 2 patients were taking neuroleptic medication, it was not possible to examine the memory effect of these drugs. In a recent review of the impact of medication on cognition, Goldberg and Gold (1995) came to the conclusion that this impact is small and that there is little reason to believe that the recent work documenting memory impairment in schizophrenia patients is the result of a negative treatment effect. Although it cannot be totally excluded that drugs have contributed to the memory dysfunction observed in this study, it is unlikely that they can account for the whole deficit.

The context-memory deficit hypothesis predicts that schizophrenic patients would be more impaired at recognition memory for spatial context information than at recognition memory for target information. This prediction was confirmed by the demonstration of a disproportionate performance deficit in the location memory task, where performance was close to chance. This clearly shows that schizophrenic patients have an impaired memory for spatial context. In contrast, they did not exhibit a disproportionate deficit in the relocation task, which was intended to assess another form of memory for spatial information. The reasons patients were disproportionately impaired in the location memory task but not in the relocation task deserve comment. Although both tasks investigated the ability to associate target and spatial information, they differ in a major aspect. Whereas in the former, participants could only perform on the basis of their associative spatial memory, in the latter, they could also perform on the basis of a nonassociative spatial memory; their response could have been based on recognition memory for the locations that were, or were not, previously occupicd by words, irrespective of which particular words occupied particular locations. The fact that patients with schizophrenia were not disproportionately impaired in the relocation task, in conditions where their associative spatial memory was defective, strongly suggests that they used a nonassociative spatial memory in the relocation task to compensate for their associative spatial memory dcficit. This interpretation is further supported by the demonstration of a significant correlation between relocation performance and the visuopattern span in the schizophrenic group and between relocation performance and the visual memory score of the WMS-R in the control group; these correlations provide evidence that performance in the relocation task relied heavily on visuospatial information processing. Taken together, these results suggest that spatial information memory per se was not impaired, but rather that the ability to bind target and spatial information was defective. However, caution is required when interpreting these results, because they were obtained using a matching procedure that excluded patients with severe memory disorders. It must therefore be asked whether this interpretation, which is based on results from patients with schizophrenia with moderately severe memory deficits, can be generalized to patients with more marked deficits. Such a generalization could be attempted on the basis of the significant correlation observed between spatial memory performance and long-term memory deficit, as measured by the delayed recall score on the WMS-R: the more severely impaired the long-term memory, the more defective the associative spatial memory. But there are also some indications that the most severely impaired patients behaved differently in the relocation task because unlike other patients, they performed at chance. Following the previous line of reasoning, this could mean that these patients did not develop compensatory strategies that were based on the use of a nonassociative form of spatial memory; alternatively, both associative and nonassociative forms of spatial memory might have been defective. Further studies using a different matching procedure are needed to investigate whether schizophrenic patients are heterogeneous as regards their memory deficit.

Whether the pattern of memory dysfunction observed in schizophrenic patients resembles that observed in patients with organic amnesia is a matter of debate (Duffy \& O'Carroll, 1994; McKenna et al., 1990; Tamlyn et al., 1992). It is unclear whether amnesia patients are disproportionatcly impaired in spatial memory tasks, with onc study reporting such an impairment (Shoqcirat \& Mayes, 1991), but not others (e.g., Hirst \& Volpe, 1984). Any conclusion therefore seems premature, even though there is evidence that amnesia patients might differ at least from schizophrenia patients with moderate memory deficit insofar as they exhibit a nonassociative spatial memory defect (Shoqeirat \& Mayes, 1991). Spatial memory, as assessed by the ability to remember locations of toys on a grid, has been found to remain intact in patients with frontal lobe excisions (Smith \& Milner, 1981). This finding has been replicated using a slightly different spatial memory task (Kohl \& Brandt, 1985). In fact, the memory dysfunction profile observed in schizophrenia patients resembles more that observed in amnesia patients with superimposed frontal lobe lesions, that is, patients with alcoholic Korsakoff's syndrome (Schacter, 1987), suggesting that the frontal lobe dysfunction associated with schizophrenia (Weinberger, 1988; Winn, 1994) plays a role in the memory dysfunction.

More direct evidence from the present study supports this view. Patients performed significantly worse than controls in tasks sensitive to frontal lobe dysfunction, such as the WCST and fluency tasks and, in agreement with Cohen and ServanSchrciber (1992), in the Stroop task. Moreover, patients' performance in the location memory task was significantly correlated with performance in the Stroop task as measured by the number of errors in the interference condition, suggesting that the disproportionate deficit of associative spatial memory was related to frontal dysfunction. This is consistent with the significant correlation between spatial memory and fluency tasks observed in the control group. However, this suggestion must be regarded as tentative because the correlations with performance in the WCST and fluency tasks were not statistically significant in the schizophrenia group.

The suggestion that frontal lobe dysfunction plays a role in the disproportionate deficit of associative spatial memory provides some clues to the functional mechanisms that under- 
lie the deficit. It has been suggested that frontal regions are involved in elaborative, strategic processes that control the segmentation and organization of ongoing experience into distinctive units that can be discriminated from one another (Fuster, 1980). The failure to organize events would make it difficult to discriminate between them with respect to features such as space. This is reminiscent of what has been described in schizophrenia patients, who fail to organize and elaborate ongoing experience (Koh et al., 1976; Russel \& Beekhuis, 1976; Russel, Bannatyne, \& Smith, 1975; Traupmann, Berzofsky, \& Kesselman, 1976). According to this view, moderately memory-impaired schizophrenic patients would be able to remember isolated aspects of recent events, such as target information, which falls at the focus of attention, as well as spatial contextual information, which falls at the periphery of attention. However, their ability to establish the links between target and contextual information, which makes it possible to identify events as separate and specific entities, would be impaired. It remains to be seen whether this impairment of associations is the result of a failure to initiate elaborative processing, or corresponds to defective elaboration per se. Finally, it is possible that schizophrenic patients also have an impairment of the storage or retrieval of associations between target and context.

This analysis points to a broader defect of elaborative processes as the cause of the disproportionate impairment of associative spatial memory. Such functional mechanisms have also been put forward to account for the impairment of conscious recollection (Huron et al., 1995) and of temporal context memory (Rizzo et al., 1996) associated with schizophrenia. It should be noted that the impairment of temporal context memory, as measured by a recency discrimination task, was observed in the same sample of patients with schizophrenia as that used in the present study, and was not significantly correlated with performance in the spatial memory tasks. This suggests that, in addition to the impairment of elaborative processing, the impairment of another type of processing might have contributed to the context memory deficit. For instance, it has been suggested that nonschizophrenic individuals can encode and retrieve spatial information not only on the basis of an elaborative information processing, which is effortful, but also on the basis of a relatively automatic activation (Hasher \& Zacks, 1979), on which task instructions, practice, and age have little or no influence (Ellis, 1990, 1991; Ellis, Kats, \& Williams, 1987; Ellis, Woodley-Zanthos, \& Dulaney, 1989). The possibility cannot be excluded that the spatial memory deficit of schizophrenia patients is due, at least in part, to an impairment of such automatic processing.

To conclude, this study provides evidence to support the context-memory deficit hypothesis of the memory impairment associated with schizophrenia. It is suggested that the disproportionate deficit of spatial context memory observed in patients with schizophrenia is related to frontal lobe dysfunction and may be part of a broader impairment of the ability to organize and elaborate information. Memory for spatial information was investigated using words presented in locations. It is not clear whether the demonstration of a defect of this kind of spatial information, which concerns local features of spatial position, can be extended to more global spatial information about the occurrence of an event. Neverthcless, the results are likcly to have implications for the psychopathology of schizophrenia because it is context memory that mediates memory for existence and identity in subjective time (Tulving, 1985) and makes memory goal directed (Moscovitch, 1992).

\section{References}

American Psychiatric Association. (1987). Diagnostic and statistical manual of mental disorders (3rd ed., rev.). Washington, DC: Author.

Andreasen, N. C. (1983a). Scale for the assessment of negative symptoms $(S A N S)$. Iowa City, University of Iowa.

Andreasen, N. C. (1983b). Scale for the assessment of positive symptoms (SAPS). lowa City, University of Iowa.

Baddeley, A. D. (1982). Domains of recollection. Psychological Review, $89,708-729$.

Baddeley, A. D. (1987). The cognitive psychology of everyday life. British Joumal of Experimental Psychology, 72, 257-269.

Bauman, E., \& Murray, D. J. (1968). Recognition versus recall in schizophrenia. Canadian Journal of Psychology, 22, 18-25.

Bentall, R. P., Baker, G. A., \& Havers, S. (1991). Reality monitoring and psychotic hallucinations. British Journal of Clinical Psychology, $30,213-222$.

Britton, P. G., \& Savage, R. D. (1966). A short form of the WAIS for use with the aged. British Joumal of Psychiatry, 112, 417-418.

Bruyer, R., \& Tuyumbu, B. (1981). Le test de fluence verbale dans une population belge d'expression française [Test of verbal fluency in a French-speaking Belgian population]. Revue de Psychologie Appliquée, 31, 1-11.

Calev, A. (1984). Recall and recognition in chronic nondemented schizophrenics: Use of matched tasks. Joumal of Abnormal Psychology, 93, 172-177.

Chouinard, G., \& Denis, J. F. (1980). Les neuroleptiques [The neuroleptics]. In P. Lalonde \& G. Grünberg (Eds.), Psychiatrie clinique: Approche contemporaine (pp. 723-742). Chicoutimi, Quibec, Canada: Gaètan Morin.

Cohen, J. D., \& Servan-Schreiber, D. (1992). Context, cortex and dopamine: A connectionist approach to behavior and biology in schizophrenia. Psychological Review, 99, 45-77.

Crawford, J. R., Allan, K. M., \& Jack, A. M. (1992). Short form of the UK WAIS-R: Regression equations and their predictive validity in a general population sample. British Joumal of Clinical Psychology, 31, 191-202.

Duffy, L., \& O'Carroll, R. (1994). Memory impairment in schizophrenia. A comparison with that observed in the Alcoholic Korsakoff Syndrome. Psychological Medicine, 24, 155-165.

Ellis, N. R. (1990). Is memory for spatial location automatically encoded? Memory and Cognition, 18, 584-592.

Ellis, N. R. (1991). Automatic and effortful processes in memory for spatial location. Bulletin of the Psychonomic Society, 29, 28-30.

Ellis, N. R., Kats, E., \& Williams, J. E. (1987). Developmental aspects of memory for spatial location. Joumal of Experimental Children Psychology, 44, 401-412.

Ellis, N. R., Woodley-Zanthos, P., \& Dulaney, C. (1989). Memory for spatial location for children, adults and mentally retarded persons. American Joumal of Mental Retardation, 93, 521-526.

Fuster, J. M. (1980). The prefrontal cortex: Anatomy, physiology and neuropsychology of the prefrontal lobe. New York: Raven Press.

Gold, J. M., Randolph, C., Carpenter, C. J., Goldberg, T. E., \& Weinberger, D. R. (1992). Forms of memory failure in schizophrenia. Joumal of Abnomal Psychology, 101, 487-494.

Goldberg, T. E., \& Gold, J. M. (1995). Neurocognitive functioning in patients with schizophrenia. In F. E. Bloom \& D. J. Kupfer (Eds.), Psychopharmacology: The fourth generation of progress (pp. 12451257). Raven Press, Ltd., New York.

Goldberg, T. E., Saint-Cyr, J. A., \& Weinberger, D. R. (1990). 
Assessment of procedural learning and problem solving in schizophrenic patients by tower of Hanoi type tasks. Journal of Neuropsychology and Clinical Neurosciences, 2, 165-173.

Goldberg, T. E., Weinberger, D. R., Pliskin, N. H., Berman, K. F., \& Podd, M. H. (1989). Recall memory deficit in schizophrenia. A possible manifestation of prefrontal dysfunction. Schizophrenia Research, 2, 251-257.

Gras-Vincendon, A., Danion, J. M., Grange, D., Bilik, M., WillardSchroeder, D., Sichel, J. P., \& Singer, L. (1994). Explicit memory, repetition priming and cognitive skill learning in schizophrenia. Schizophrenia Research, 13, 117-126.

Guy, W. (Ed.) (1976). ECDEU assessment manual for psychophannacology (Publication ADM 76-338). Washington, DC: U.S. Department of Health, Education, and Welfare.

Hasher, L., \& Zacks, R. T. (1979). Automatic and effortful processes in memory. Journal of Experimental Psychology: General, 108, 356388.

Hirst, W., \& Volpe, B. T. (1984). Encoding of spatial relations with amnesia. Neuropsychologia, 22, 631-634.

Huron, C., Danion, J. M., Giacomini, F., Grangé, D., Robert, P., \& Rizzo, L. (1995). Impairment of recognition memory with, but not without, conscious recollection in schizophrenia. American Joumal of Psychiatry, 12, 1737-1742.

Huston, P. E., \& Shakow, D. (1949). Learning capacity in schizophrenia: With special reference to the concept of deterioration. Ameri can Journal of Psychiatry, . 105, 881-888.

Jacoby, L. L., \& Dallas, M. (1981). On the relationship between autobiographical memory and perceptual learning. Journal of Experimental Psychology: General, 3, 306-340.

Koh, S. D., Kayton, L., \& Peterson, R. A. (1976). Affective encoding and consequent remembering in schizophrenic young adults. Joumal of Clinical Psychology, 42, 879-887.

Kohl, D., \& Brandt, J. (1985). An Automatic encoding deficit in the amnesia of Korsakoff's syndrome. Annals of the New York Academy of Sciences, 444, 460-462.

Kornetsky, C., Pettit, M., \& Wynne, R. (1959). A comparison of the psychological effects of acute and chronic administration of chlorpromazine and secobarbital (quinalbarbitonc) in schizophrenic patients. Journal of Mentul Sciences, 105, 190-198.

Mandler, G. (1980). Recognizing: The judgement of previous occurrence. Psychological Review, 87, 252-271.

Mayes, A. R., Meudell, P., \& McDonald, C. (1991). Disproportionate intentional spatial-memory impairments in amnesia. Neuropsychologia, 29, 771-784.

McKenna, P. J., Tamlyn, D., Lund, C. E., Mortimer, A. M., Hammond, S., \& Baddeley, A. D. (1990). Amnesic syndrome in schizophrenia. Psychological Medicine, 20,967-972.

Milner, B. (1971). Interhemispheric differences in the localization of psychological processes in man. British Medical Bulletin, 27, 272-277.

Moscovitch, M. (1992). A neuropsychological model of memory and consciousness. In L. R. Squire \& N. Butters (Eds.), Neuropsychology of memory (2nd ed., pp. 5-22). New York: Guilford Press.

Nachmani, G., \& Cohen, B. D. (1969). Recall and recognition free learning in schizophrenics. Journal of Abnormal Psychology, 74, 511-516.

Nelson, H. E. (1976). A modified card sorting test sensitive to frontal lobe defects. Cortex, 12, 313-324.

Overall, J. E., \& Gorham, D. R. (1962). The brief psychiatric rating scale. Psychological reports, 10, 799-812.

Philips, W. A. (1983). Short-term visual memory. Philosophical Transactions of the Royal Society of London, 302, 295-309.

Rizzo, L., Danion, J. M. D., Van Der Linden, M., \& Grangé, D. (1996). Schizophrenic patients remember that an event has occurred, but not when. British Joumal of Psychiatry, 168, 427-431.
Russel, P. N., Bannatyne, P. A., \& Smith, J. F. (1975). Associative strength as a mode of organization in recall and recognition: $\mathrm{A}$ comparison of schizophrenics and normals. Joumal of Abnormal Psychology, 84, 122-128.

Russel, P. N., \& Beekhuis, M. E. (1976). Organization in memory: A comparison of psychotics and normals. Journal of Abnormal Psychology, 85, 527-534.

Schacter, D. L. (1987). Implicit memory: History and current status. Joumal of Experimental Psychology: Leaming, Memory, and Cognition, 13, 501-518.

Schmand, B., Brand, N., \& Kuipers, T. (1992). Procedural learning of cognitive and motor skills in psychotic patients. Schizophrenia Research, 8, 157-170.

Schwartz, B. L., Deutsch, L. H., Cohen, C., Warden, D., \& Deutsch, S. I. (1991). Memory for temporal order in schizophrenia. Biological Psychiatry, 29, 329-339.

Schwartz, B. L., Rosse, R. B., \& Deutsch, S. I. (1993). Limits of the processing view in accounting for dissociations among memory measures in a clinical population. Memory and Cognition, 21, 63-72.

Shoqeirat, M. A., \& Mayes, A. R. (1988). Spatiotemporal memory and rate of forgetting in acute schizophrenics. Psychological Medicine, 18, $843-853$.

Shoqeirat, M. A., \& Mayes, A. R. (1991). Disproportionate incidental spatial-memory and recall deficits in amnesia. Neuropsychologia, 29, $749-769$.

Simpson, G. M., \& Angus, J. W. S. (1970). A rating scale for extrapyramidal side effects. Acta Psychiatrica Scandinavica, 212(Suppl. 220), 11-19.

Smith, M. L., \& Milner, B. (1981). The role of the right hippocampus in the recall of spatial location. Neuropsychologia, 19, 781-794.

Stroop, J. R. (1935). Studies of interference in serial verbal reactions. Joumal of Experimental Psychology, 18, 643-662.

Tamlyn, D., McKenna, P. J., Mortimer, A. M., Lund, C. E., Hammond, S., \& Baddeley, A. D. (1992). Memory impairment in schizophrenia: Its extent, affiliations and neuropsychological character. Psychological Medicine, 22, 101-115.

Traupmann, K. L., Berzofsky, M., \& Kesselman, M. (1976). Encoding or taxonomic word categories by schizophrenics. Joumal of Abnormal Psychology, 85, 350-355.

Tulving, E. (1985). Memory and consciousness. Canadian Psychologist, 26, 1-12.

Wechsler, D. (1981). Manual for the Wechsler Adult Intelligence ScaleRevised. New York: Psychological Corporation.

Wechsler, D. (1989). [French translation and adaptation of the Wechsler Adult Intelligence Scale-Revised] Paris: Centre de Psychologie Appliquée.

Weinberger, D. R. (1988). Schizophrenia and the frontal lobe. Trends in Neurosciences, 11, 367-370.

Wilson, B., Cockburn, J., Baddeley, A., \& Hiorns, R. (1989). The development and validation of a test battery for detecting and monitoring everyday memory problems. Joumal of Clinical and Experimental Neuropsychology, 11, 855-870.

Wilson, L., Scott, J. H., \& Power, K. G. (1987). Developmental differences in span of visual memory for pattern. British Journal of Experimental Psychological Society, 5, 249-255.

Winn, P. (1994). Schizophrenia research moves to the prefrontal cortex. Trends in Neurosciences, 17, 265-268.

Received June 2, 1995

Revision received October 17, 1995 Accepted November 17, 1995 